\title{
Comparison of Oral Findings of Common Variable Immune Deficiency and Nephrotic Syndrome Patients with Hy- pogammaglobulinemia to Healthy Individuals
}

\author{
Hulya Cakir Karabas ${ }^{1 *}$ (D) Ilknur Ozcan ${ }^{1}$ (D) Ozlem Guler ${ }^{\text {(D) }}$, Muhlis Cem Ar ${ }^{3}$ (D) Arif \\ Atahan Cagatay ${ }^{4}$ (D) and Aydin Turkmen ${ }^{5}$ (iD
}

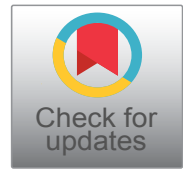

${ }^{1}$ Faculty of Dentistry, Department of Oral and Maxillofacial Radiology, Istanbul University, Turkey

${ }^{2}$ Faculty of Medicine, Department of Infectious Diseases and Clinical Microbiology, Kocaeli University, Turkey

${ }^{3}$ Faculty of Medicine, Department of Internal Medicine, Division of Hematology, Istanbul University - Cerrahpasa, Turkey

${ }^{4}$ Faculty of Medicine, Department of Infectious Diseases and Clinical Microbiology, Istanbul University, Turkey

${ }^{5}$ Faculty of Medicine, Division of Nephrology, Department of Internal Medicine, Istanbul University, Turkey

*Corresponding author: Hulya Cakir Karabas, Faculty of Dentistry, Department of Oral and Maxillofacial Radiology, Istanbul University, Prof. Dr. Cavit Orhan Tütengil Sokak. No: 4 Vezneciler-Fatih-Istanbul, Turkey, E-mail: hulya.cakirkarabas@gmail.com

\begin{abstract}
Purpose: A group of disorders featuring lower serum immunoglobulin levels is called hypogammaglobulinemia. Being a primary immune disorder, Common variable immunodeficiency (CVID) is characterized by primary hypogammaglobulinemia. Nephrotic syndrome (NS) is an entity associated with secondary hypogammaglobulinemia. Lower concentrations of immunoglobulins in saliva may attenuate oral health. The aim of this study is to investigate the impact of salivary immunoglobulin concentrations on oral health in patients with primary and secondary hypogammaglobulinemia.
\end{abstract}

Materials and methods: This study was a case-control study involving 19 CVID and 19 NS patients compared to 37 immunologically healthy individuals. Total saliva $\operatorname{lgA}$, IgM and IgG levels, saliva microbiological parameters (total streptococci, mutans streptococci, lactobacilli, and total anaerobic bacteria counts), intraoral mucosal lesions, panoramic and bitewing radiographies, decayed, missing and filled teeth (DMFT), decayed, missing and filled surfaces (DMFS), plaque index, bleeding on probing (BOP) were analyzed.

Results: The total concentrations of salivary lgG were statistically significant lower $(p<0.0001)$ in the case group. However, no such difference existed in total $\lg \mathrm{A}$ and $\lg \mathrm{M}$ levels. With regard to DMFT and DMFS indices, plaque index, or BOP, no major differences were found between the case and the control group. The mean colony-forming unit numbers of salivary lactobacilli, total streptococci, and anaerobic flora were significantly lower $(p<0.001)$ in the case group.

Conclusion: Although the mean IgG level was lower among immunodeficient patients, the pathological oral data showed no significant difference. This may be explained by the least important effect of $\lg G$ on oral health compared to $\lg A$ and $\lg \mathrm{M}$.

\section{Keywords}

Hypogammaglobulinemia, Common variable immunodeficiency, Nephrotic syndrome, Oral findings, Oral health

\section{Introduction}

Immunoglobulins, secreted by the B-lymphocytes, are $\mathrm{Y}$-shaped proteins consisting of two pairs of identical light and heavy polypeptide chains. The structure of the heavy chain defines the type of the immunoglobulin as $\lg A$, IgG, IgM, IgD, or IgE [1-4]. They play crucial roles in immunity by neutralizing and opsonizing the antigens via direct binding and stimulating cytokine and chemokine release to further induce specific immune response [4].

Hypogammaglobulinemia is the common name used

Citation: Karabas HC, Ozcan I, Guler O, Ar MC, Cagatay AA, et al. (2020) Comparison of Oral Findings of Common Variable Immune Deficiency and Nephrotic Syndrome Patients with Hypogammaglobulinemia to Healthy Individuals. Int J Oral Dent Health 6:116. doi.org/10.23937/2469-5734/1510116 Accepted: September 26, 2020: Published: September 28, 2020

Copyright: (c) 2020 Karabas HC, et al. This is an open-access article distributed under the terms of the Creative Commons Attribution License, which permits unrestricted use, distribution, and reproduction in any medium, provided the original author and source are credited. 
for a group of disorders featuring lower serum immunoglobulin levels. It can be classified as primary or secondary and has been affiliated with various etiologies. Primary hypogammaglobulinemia is a genetic disorder and involves diseases like common variable immunodeficiency (CVID) [5]. CVID is a primary immune disorder which shows heterogenous clinical features and characterized by hypogammaglobulinemia leading to recurrent bacterial infections primarily affecting the respiratory tract like sinusitis, bronchitis and pneumoniae $[4,6,7]$. Patients are usually treated with regular intravenous immunoglobulin injections every 3-4 weeks [4].

Secondary or acquired hypogammaglobulinemia has been reported to result from several medical conditions including malignancies, infectious and/or metabolic disorders, medications like glucocorticosteroids and immunomodulatory drugs, environmental exposures, and malnutrition [5]. Nephrotic syndrome (NS) is another entity associated with secondary hypogammaglobulinemia. It is a clinical condition that includes proteinuria, peripheral oedema, hypoalbuminaemia and hypercholesterolaemia [8]. Patients with NS are at high risk for infections, in part, due to urinary loss of immunoglobulins and complement proteins via kidneys $[9,10]$.

Immunoglobulins are secreted into the saliva and have been associated with maintenance of oral health. Lower concentrations of immunoglobulins in saliva may attenuate oral health leading to diseases in the oral cavity [5].

Aim of this study is to investigate the impact of salivary immunoglobulin (IgA, IgG and IgM) concentrations on oral health and content of normal oral flora in patients with primary and secondary hypogammaglobulinemia.

\section{Materials and Methods}

Hypogammaglobulinemia is defined as a plasmatic level of immunoglobulin (Ig) under $5 \mathrm{~g} / \mathrm{L}$ [11]. Our study was designed as a case-control study to detect oral pathologies among patients with primary and secondary hypogammaglobulinemia. Immunoglobulins in saliva are affected by many factors and their levels in saliva are directly associated with mucosal permeability; therefore, in this study, their saliva levels were measured instead of serum [12]. We analyzed total saliva IgA, IgM and IgG levels, clinical data of dental caries, periodontal conditions and mucosal lesions, additionally saliva microbiological parameters (total streptococci, mutans streptococci, lactobacilli and total anaerobic bacteria counts). All participants signed an informed consent form after written and verbal information was provided with regards to details and the aim of present study. Out of 38 patients included in this study, 19 of them were diagnosed with CVID and other half was diagnosed with NS. The patients were examined at outpatient clinical ward in Department of Internal Diseases to exclude additional systemic diseases which might cause any oral disease or affect flow and quality of the saliva. Hypogammaglobulinemia was verified by serum electrophoresis of the patients. The control group included 37 age and sex matched immunologically healthy subjects.

Prior to oral examination, saliva was collected from each patient. Patients had not eaten, drunk or smoked for 1 hour before obtaining saliva. For microbiological analysis 100 microliter of saliva was transferred to a tube containing $1 \mathrm{ml}$ of tryptic soy broth (bioMérieux, France) (TSB) supplemented with $20 \%$ glycerol. In order to detect levels of $\lg A$, IgG and IgM levels in the rest of saliva for nephelometric analysis, all samples have been stored at $-20{ }^{\circ} \mathrm{C}$ until it was analyzed [13]. The clinical intraoral examination of each individual was performed by a trained dentist under artificial light using a mouth mirror, explorer and a periodontal probe under standard conditions. Intraoral mucosal lesions, panoramic and bitewing radiographies, decayed, missing and filled teeth (DMFT), decayed, missing and filled surfaces (DMFS), plaque index, bleeding on probing (BOP) were recorded.

TBS tubes were thawed and vortexed thoroughly for a minute for microbiological analysis. After serial 10-fold dilutions to detect bacteria, saliva was plated as follows: For total streptococci and mutans streptococci (MS) on Mitis salivarius (Becton Dickinson, USA) and Mitis salivariusbacitracin agar (Becton Dickinson, USA) plates, respectively. The Mitis salivarius agar plates were supplemented with $20 \%$ sucrose and incubated aerobically for 2 days in $37{ }^{\circ} \mathrm{C}$. The Mitis salivarius bacitracin agar plates were supplemented with both sucrose and 0.5 $\mu \mathrm{g} / \mathrm{ml}$ bacitracin (Sigma-Aldrich, USA) and MS were incubated for 3 days in a $7 \% \mathrm{CO}_{2}$ atmosphere at $37^{\circ} \mathrm{C}$. For Lactobacilli saliva were cultivated on Rogosa SL (Becton Dickinson, USA) agar plates and incubated anaerobically for 3 days at $37^{\circ} \mathrm{C}$. The total flora was determined by plating samples on blood agar plates containing $5 \%$ bovine blood (bioMérieux, France) and incubated anaerobically for 2 days at $37^{\circ} \mathrm{C}$. After appropriate incubation times the bacterial number of colony forming units (CFU/ml saliva) was determined [13].

All rest of saliva samples were thawed and enzyme immunoassay kits (Eastbiopharm, Hangzou, China) was used for the assay for quantitative measurement of IgA, IgM, IgG in saliva samples. Saliva samples were added to monoclonal antibody enzyme wells which was pre-coated with anti-human IgA, anti-human IgG, anti-human IgM monoclonal antibody. After incubation IgA, IgG, IgM antibodies labeled with biotin and combined with Streptavidin-HRP to form immune complex; then carry out incubation and washing again to remove the uncombined enzyme. Then, Chromogen Solution A, $B$ were added for the color of the liquid changes into the blue. And at the effect of acid, the color finally becomes 
Table 1: Age and oral findings of participants.

\begin{tabular}{|l|l|l|l|l|l|}
\hline & \multicolumn{2}{l}{ Groups Mean \pm SD } & \multicolumn{2}{l|}{ post-hoc } \\
\hline & Control $(\mathbf{n}=\mathbf{3 7})$ & CVID $(\mathbf{n}=\mathbf{1 9})$ & NS (n=19) & p & 0.684 \\
\hline Age & $37 \pm 16$ & $35 \pm 13$ & $42 \pm 19$ & 0.081 \\
\hline DMFT & $6 \pm 7$ & $11 \pm 9$ & $9 \pm 10$ & 0.107 \\
\hline DMFS & $25 \pm 31$ & $43 \pm 44$ & $35 \pm 46$ & 0.333 \\
\hline Plaque index & $65 \pm 30$ & $61 \pm 32$ & $53 \pm 34$ & 0.908 \\
\hline BOP & $57 \pm 31$ & $57 \pm 30$ & $54 \pm 36$ & \\
\hline
\end{tabular}

Table 2: Total antibodies in saliva of immunodeficient patients and controls.

\begin{tabular}{|l|l|l|l|l|l|}
\hline & \multicolumn{2}{l|}{ Groups Mean \pm SD } & \multicolumn{2}{l|}{ Significance } \\
\cline { 2 - 6 } & Control $(\mathbf{n}=\mathbf{3 7})$ & CVID $(\mathbf{n}=\mathbf{1 9})$ & NS $(\mathbf{n}=\mathbf{1 9})$ & p & post-hoc \\
\hline Saliva IgG & $256.88 \pm 103.22$ & $171.50 \pm 88.07$ & $140.50 \pm 96.90$ & $<0.001$ & $1-2,1-3$ \\
\hline Saliva IgA & $0.82 \pm 0.34$ & $0.70 \pm 0.39$ & $0.77 \pm 0.46$ & 0.519 \\
\hline Saliva IgM & $1.04 \pm 0.31$ & $0.86 \pm 0.52$ & $0.95 \pm 0.35$ & 0.738 \\
\hline
\end{tabular}

Table 3: Salivary microbiological data of participants.

\begin{tabular}{|c|c|c|c|c|c|}
\hline & \multicolumn{5}{|c|}{ Groups Mean \pm SD } \\
\hline & Control $(n=37)$ & CVID (n=19) & NS ( $n=19)$ & $\mathbf{p}$ & post-hoc \\
\hline Total streptococi & $48649 \pm 28103$ & $9842 \pm 13446$ & $31684 \pm 23884$ & $<0.001$ & $1-2,2-3$ \\
\hline Mutans streptococci & $16159 \pm 13671$ & $3147 \pm 6896$ & $10763 \pm 13066$ & 0.001 & $1-2$ \\
\hline Lactobacillus & $24730 \pm 16668$ & $3537 \pm 5890$ & $16184 \pm 16534$ & $<0.001$ & $1-2,2-3$ \\
\hline Total anaerop & $66486 \pm 23240$ & $28053 \pm 17853$ & $56842 \pm 27899$ & $<0.001$ & $1-2,2-3$ \\
\hline
\end{tabular}

yellow. Optical density was read on a standard automated plate reader at $450 \mathrm{~nm}$ (Perkin Elmer).

\section{Statistical analysis}

All statistical calculations were performed with the Statistical Package for Social Sciences (version 11.0 for Windows; SPSS, Inc., Chicago, IL). Data was presented as mean, standard deviation (SD), median, minimum, maximum, percentage and numbers. Shapiro Wilk test was used to evaluate distribution of variables. Kruskal-Wallis test was used because data showed non-normal distribution. Comparison of groups was calculated via post hoc tests and categorical variables were compared with groups via Chi-Square test. $P<0.05$ was considered to denote a significant difference.

\section{Results}

A total of 75 (19 CVID, 19 NS and 37 control group) participants were included. The mean ages of the participants were shown on Table 1. The total concentrations of salivary IgG were statistically significant lower $(p<$ 0.0001 ) in immunodeficient patients than in the controls (Table 2). However, no such difference existed in total IgA and IgM levels. The mean colony forming unit numbers of salivary lactobacilli, total streptococci and anaerobic flora were significantly lower in immunodeficient group compared to control group $(p<0.001)$. CVID group and NS group also demonstrated statistically significant differences, in which the total colony forming unit numbers were the lowest in CVID group $(p<0.001)$.

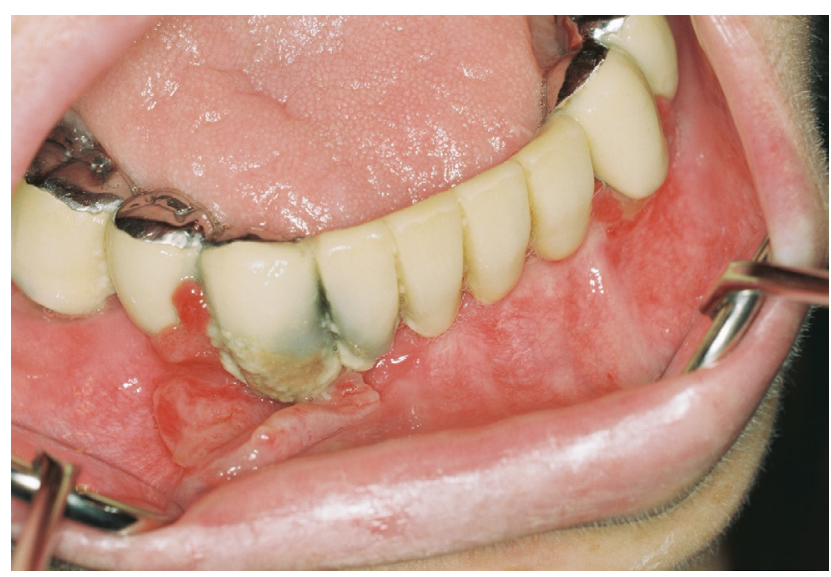

Figure 1: Inflammatory gingival hyperplasia with CVID patient.

The total numbers of salivary mutans streptococci was lower in CVID group compared to control group ( $p=$ 0.001 ) but NS group and control group didn't have significant difference (Table 3). No major differences were found between the immunodeficient patients and the control group with regards to DMFT and DMFS indices, plaque index, or BOP (Table 1). Mucosal lesions were not detected at any patient, but inflammatory gingival hyperplasia was detected in 1 patient with CVID (Figure 1).

\section{Discussion}

IgA, IgG and IgM are present at saliva and main immunoglobulin type at secretions is IgA $[14,15]$. Although 
we know more about oral microbial ecology and the role of immunoglobulins, we are less aware of the oral consequences of antibody deficiencies. Oral healths of individuals with hypogammaglobulinemia have rarely been studied. The reported main oral features of these patients involve periodontal disease, recurrent aphthous ulcer (RAU), lichenoid lesions, enamel hypoplasia and pseudomembranous candidiasis [5]. Also necrotizing periodontal diseases have been reported [16].

$\lg A$ is the predominant immunoglobulin isotype in human mucosal secretion. Measurement of total salivary IgA provides a measure of the integrity of the human mucosal immune system [12]. Salivary IgA has important immune roles for oral cavity. But, the relationship between low IgA and periodontal diseases-dental caries risks is controversial; some studies showed a relationship [15] whereas others did not prove it [17]. Salivary IgA secreted from plasma cells in salivary glands, salivary IgG mainly delivered from serum. As salivary IgG's source is serum and in CVID-NS patients show lack of IgG's in serum, it is a predictable outcome that salivary IgG levels decrease [18]. IgA is actively secreted from salivary glands and major part of the secretion comes from parotid [18], it seems that low levels of serum IgA levels do not affect the salivary levels of IgA. IgG plays a small part in the immune defense of mucosa due to absence of receptors in the secretory epithelial cell enabling its transcytosis [19]. Even though the mean IgG level was lower among immunodeficient patients, the pathological oral data showed no significant difference. This situation may be explained the least importance effect of IgG for oral health compared to IgA and IgM [20]. As dental caries development is a multifactorial process, not only immunologic factors but also patients' habits about nutrition (frequent consumption of refined carbohydrates), poor oral hygiene, genetic factors, salivary flow and composition, number of cariogenic bacteria etc. have role on dental caries development [21]. Therefore, the role of immunoglobulins at caries development is a controversial issue [20]. Our study showed that besides the different salivary levels of IgG between control and CVID, and also control and NS patients, there is no inclination to dental caries and periodontal problems in CVID/NS patients. This finding is similar to Kirstila, et al. results [13]. Consequently, our results support the idea of IgG's role on caries development is low or absent.

Mutans streptococci and lactobacilli are correlated to dental caries. Conversely, total anaerobic mouth flora is found more abundant among caries-free individuals [22]. In our study, salivary lactobacilli, total streptococci, mutans streptococci and anaerobic flora numbers were lower among immunodeficient patients compared to control group on the contrary to the literature [13]. This situation may be explained by bad oral hygiene of the control group and the fact that both CVID and NS patients were under regular medical control. Although none of the patients used antibiotics during the saliva collection period, incidence of antibiotic usage is higher in immunodeficient patients [23]. It also may be the cause of low numbers of microorganisms.

Oral symptoms of CVID are reported as gingivitis, lichenoid reactions and necrotizing ulcerative periodontitis [24]. None of our patients showed oral lesions except one patient with inflammatory gingival hyperplasia. Gingivitis is a common condition in patients participating in our study, with bleeding index's median value is $59 \%$, $57 \%, 46 \%$ for control, CVID and NS patients respectively. There was no statistically significant difference between groups about gingival inflammation and this finding is coherent with Kirstila, et al. findings [13].

To sum up, although our study did not prove a relationship between hypogammaglobulinemia and oral health of the patients, careful oral health monitoring is essential for them since they are more susceptible to respiratory infections due to the presence of various respiratory pathogens in the oral cavity [25].

\section{Acknowledgements}

This study was supported by the Istanbul University Scientific Research Projects (Project number: 17746), Turkey. The authors declare that there are no conflicts of interest in relation to this study.

\section{References}

1. Trochimiak T, Hübner-Woźniak E (2012) Effect of exercise on the level of immunoglobulin a in saliva. Biol Sport 29: 255-261.

2. Giuca MR, Pasini M, Tecco S, Giuca G, Marzo G (2014) Levels of salivary immunoglobulins and periodontal evaluation in smoking patients. BMC Immunol 15: 5.

3. Cipe FE, Dogu F, Guloglu D, Aytekin C, Polat M, et al. (2013) B-cell subsets in patients with transient hypogammaglobulinemia of infancy, partial IgA deficiency, and selective IgM deficiency. J Investig Allergol Clin Immunol 23: 94-100.

4. Rose ME, Lang DM (2006) Evaluating and managing hypogammaglobulinemia. Cleve Clin J Med 73: 133-144.

5. Fernandes KS, Kokron CM, Barros MT, Kalil J, Gallottini M (2012) Oral manifestations in patients with hypogammaglobulinemia. Oral Surg Oral Med Oral Pathol Oral Radiol 114: $19-24$

6. Cunningham-Rundles C, Bodian C (1999) Common variable immunodeficiency: Clinical and immunological features of 248 patients. Clin Immunol 92: 34-48.

7. Yong PF, Tarzi M, Chua I, Grimbacher B, Chee R (2008) Common variable immunodeficiency: An update on etiology and management. Immunol Allergy Clin North Am 28: 367-386.

8. Hull RP, Goldsmith DJ (2008) Nephrotic syndrome in adults. BMJ 336: 1185-1189.

9. Keddis MT, Karnath BM (2007) The nephrotic syndrome. Hospital physician 43: 25-30.

10. Seigneux de S, Martin PY (2009) Management of patients with nephrotic syndrome. Swiss Med Wkly 139: 416-422. 
11. Samson M, Audia S, Lakomy D, Bonnotte B, Tavernier C et al. (2011) Diagnostic strategy for patients with hypogammaglobulinemia in rheumatology. Joint Bone Spine 78: 241-245.

12. Gleeson M, Cripps AW, Clancy RL (1995) Modifiers of the human mucosal immune system. Immunol Cell Biol 73: 397-404.

13. Kirstilä V, Tenovuo J, Ruuskanen O, Nikoskelainen J, Irjala K, et al. (1994) Salivary defense factors and oral health in patients with common variable immunodeficiency. J Clin Immunol 14: 229-236.

14. Patricia de DVA, Grégio AM, Machado MA, Lima de AA, Azevedo LR (2008) Saliva composition and functions: A comprehensive review. J Contemp Dent Pract 9: 72-80.

15. Jafarzadeh A, Sadeghi M, Karam GA, Vazirinejad R (2010) Salivary IgA and IgE levels in healthy subjects: Relation to age and gender. Braz Oral Res 24: 21-27.

16. Dalla Torre D, Burtscher D, Jank S, Kloss FR (2012) Necrotizing periodontitis as a possible manifestation of common variable immunodeficiency. Int J Oral Maxillofac Surg 41: 1546-1549.

17. Fernandes KS, Lima MB, Martins CP, Maria CS, Fabio DN et al. (2016) Salivary immunoglobulins in individuals with common variable immunodeficiency. Braz Dent J 27: 641645.
18. Kaufman E, Lamster IB (2000) Analysis of saliva for periodontal diagnosis-a review. J Clin Periodontol 27: 453-465.

19. Romero MR, Lozano ML, Posada C, Rueda PA, Roa NS, et al. (2011) Immunoglobulin A, G and M levels in saliva in children between 3-12 years of age, healthy and with gingivitis. Acta Odontol Latinoam 24: 176-182.

20. Nikfarjam J, Pourpak Z, Shahrabi M, Nikfarjam L, Kouhkan A, et al. (2004) Oral manifestations in selective IgA deficiency. Int J Dent Hyg 2: 19-25.

21. Selwitz RH, Ismail Al, Pitts NB (2007) Dental caries. Lancet 369: 51-59.

22. Kirstilä V, Häkkinen $P$, Jentsch H, Vilja P, Tenovuo J (1998) Longitudinal analysis of the association of human salivary antimicrobial agents with caries increment and cariogenic micro-organisms: A two-year cohort study. J Dent Res 77: 73-80.

23. Cunningham-Rundles $C$ (2010) How I treat common variable immune deficiency. Blood 116: 7-15.

24. Szczawinska-Poplonyk A, Gerreth K, Breborowicz A, Borysewicz-Lewicka M (2009) Oral manifestations of primary immune deficiencies in children. Oral Surg Oral Med Oral Pathol Oral Radiol Endod 108: 9-20.

25. Fernandes KS, Lima MB, Martins CP, et al. Salivary Immunoglobulins in Individuals with Common Variable Immunodeficiency. Braz Dent J. 2016;27(6):641-645. 